ARTIGO ORIGINAL ORIGINAL ARTICLE

\section{Custos com os cuidados da atrofia muscular espinhal 5q (AME-5q) no Brasil}

\author{
Spinal muscular atrophy (SMA-5q) care-associated costs in Brazil
}

\section{Palavras-chave:}

AME-5q, microcusteio, Time-driven Activity-based Costing, TDABC, custos em doenças raras

\section{Keywords:}

SMA-5q, microcosting, Timedriven Activity-based Costing, TDABC, rare disease costs

\section{RESUMO}

Objetivo: Este estudo estimou o custo individual de uma coorte brasileira de pacientes diagnosticados com atrofia muscular espinhal 5q (AME-5q) empregando a técnica de microcusteio considerando custos diretos e indiretos ao longo de um ano. Métodos: Foram avaliados custos hospitalares, de cuidados domiciliares e de transporte de uma coorte de pacientes em acompanhamento em 2019 no Hospital de Clínicas de Porto Alegre. O método de custeio utilizado para a avaliação de custos hospitalares foi o Time-driven Activity-based Costing (TDABC). Para os custos com cuidados domiciliares, foram realizadas entrevistas com os familiares dos pacientes. Para a estimativa de custos com transporte, valores de referência de custo por quilômetro rodado foram considerados. Os custos anuais hospitalares, domiciliares e com transporte por paciente foram calculados e estratificados de acordo com o uso ou não do medicamento nusinersena, o tipo de AME-5q e a faixa etária dos pacientes. Resultados: Foram analisados dados de 43 pacientes; 5 deles fizeram uso de nusinersena. O custo médio dos 38 pacientes que não utilizaram o medicamento foi de R\$ 4.870 (mín. R\$ 190 - máx. R\$ 67.138), enquanto o custo médio dos 5 pacientes que fizeram uso de nusinersena foi de R\$ 600.347 (mín. R\$342.679 - máx. R\$ 813.513). No grupo de pacientes que usaram nusinersena, 91\% do custo total foi devido ao medicamento. No que diz respeito ao transporte, foi observado um custo anual médio de R\$ 4.895 (mín. R\$ 18 - máx. R\$ 73.382), e o gasto médio anual familiar por paciente reportado foi de R\$32.262 (mín. R\$ 240 - máx. R\$110.952). Os pacientes com AME-5q tipos 1 ou 2 apresentaram, em média, maior custo do que aqueles com tipo 3 ou 4, bem como os pacientes menores de 6 anos de idade em relação aos demais. Conclusões: $O$ custo do manejo de pacientes com AME-5q demonstra valores expressivos tanto na perspectiva do sistema de saúde quanto das suas famílias. Essas estimativas foram diretamente relacionadas ao tipo da doença e ao uso de terapia-alvo.

\footnotetext{
ABSTRACT

Objective: This study estimated the individual cost over a year of monitoring a Brazilian cohort of patients diagnosed with spinal muscular atrophy $5 q$ ( $5 q$ SMA) using a microcosting technique, including direct and indirect costs. Methods: The hospital, home care, and transportation costs of a cohort of patients monitored at the Hospital de Clínicas de Porto Alegre were evaluated along 2019.

Recebido em: 25/03/2021. Aprovado para publicação em: 14/04/2021

1. Instituto de Avaliação de Tecnologias em Saúde/Instituto Nacional de Ciência e Tecnologia (IATS/INCT), Porto Alegre, RS, Brasil.

2. Escola Politécnica, Pontifícia Universidade Católica do Rio Grande do Sul, Porto Alegre, RS, Brasil.

3. Programa de Pós-Graduação em Epidemiologia, Universidade Federal do Rio Grande do Sul, Porto Alegre, RS, Brasil.

4. Hospital de Clínicas de Porto Alegre, Porto Alegre, RS, Brasil.

5. Neurogenética, Centro de Pesquisa Clínica, Hospital de Clínicas de Porto Alegre, Porto Alegre, RS, Brasil.

6. Programa de Pós-Graduação em Medicina: Ciências Médicas, Faculdade de Medicina, Universidade Federal do Rio Grande do Sul, Porto Alegre, RS, Brasil.

7. Departamento de Medicina Interna, Faculdade de Medicina, Universidade Federal do Rio Grande do Sul, Porto Alegre, RS, Brasil. 8. Serviço de Genética Médica, Hospital de Clínicas de Porto Alegre, Porto Alegre, RS, Brasil.

9. Serviço de Neurologia, Hospital de Clínicas de Porto Alegre, Porto Alegre, RS, Brasil.

10. Produtos Roche Químicos e Farmacêuticos S/A, São Paulo, SP, Brasil.

Financiamento: Esta pesquisa foi financiada pela empresa Produtos Roche Químicos e Farmacêuticos S/A do Brasil. O Instituto de Avaliação de Tecnologia em Saúde foi responsável pela condução do estudo, escrita do manuscrito e revisão editorial. Conflito de interesses: $O$ estudo foi uma iniciativa da empresa Produtos Roche Químicos e Farmacêuticos S/A do Brasil. Os pesquisadores envolvidos no estudo tiveram plena autonomia na condução do estudo e responsabilizam-se pelos dados aqui apresentados. Autor correspondente: Carisi Anne Polanczyk. Instituto de Avaliação de Tecnologias em Saúde/Instituto Nacional de Ciência e Tecnologia (IATS/INCT), Universidade Federal do Rio Grande do Sul (UFRGS). Rua Ramiro Barcelos, 2.350, prédio 21, sala 507, Porto Alegre, RS, Brasil. CEP: 90035-903. E-mail: cpolanczyk@hcpa.edu.br
} 
The costing method used for the evaluation of hospital costs was the Time-driven Activity-based Costing (TDABC). For the costs of home care, interviews were conducted with family members. For the estimation of transportation costs, reference values of cost per kilometer traveled were considered. Annual hospital, home, and transportation costs per patient were calculated and stratified according to the use or not of the drug nusinersen, the type of 5q SMA and the patients' age group. Results: Data from 43 patients were analyzed, of which 5 were treated with nusinersen. The average cost of the 38 patients who did not use the medication was R\$ 4.870 (min R\$190 max R\$67.138), while the average cost of the 5 patients who were treated with nusinersen was $\mathrm{R} \$ 600.347$ (min $\mathrm{R} \$ 342.679$ - $\max \mathrm{R} \$ 813.513$ ). In the group of patients who used nusinersen, $91 \%$ of the total cost was due to medication. Concerning transportation, an average annual cost of $\mathrm{R} \$ 4.895$ (min R\$ 18 - max R\$ 73.382) was observed and the average annual family expenditure per patient reported was $R \$ 32.262$ (min $R \$ 240$ - max R\$110,952). Patients with 5q SMA type 1 or 2 on average had a higher cost than those with 5 q SMA type 3 or 4, as well as patients under 6 years of age in comparison to the others. Conclusions: The cost of medical care for patients with 5q SMA is expressive from the health system's perspective and patients' families. These estimates are directly related to the type of disease and the therapy used.

\section{Introdução}

A atrofia muscular espinhal $5 q$ (AME-5q) é a causa mais frequente de morte infantil decorrente de uma condição monogênica (Araújo et al., 2005; Verhaart et al., 2017). Trata-se de um distúrbio neuromuscular autossômico recessivo raro, grave, com diferentes graus de comprometimento da função muscular e da expectativa e da qualidade de vida dos indivíduos acometidos pela doença (Lunn \& Wang, 2008). A fisiopatologia da doença é caracterizada pela disfunção e morte de neurônios motores inferiores, devido à diminuição da quantidade de proteína SMN funcional, que resulta em fraqueza e atrofia muscular progressiva (Verhaart et al., 2017; Lunn \& Wang, 2008).

Sendo uma condição neurodegenerativa progressiva, a AME-5q requer cuidados multidisciplinares e medidas de suporte e acompanhamento médicos especializados (Brasil, 2019). Recentemente foram desenvolvidas terapias avançadas específicas para o manejo da doença, e a primeira delas a ser incorporada na prática clínica nos diferentes países foi o medicamento nusinersena (aprovado em 2016 pelo Food and Drug Administration nos Estados Unidos). O nusinersena é um oligonucleotídeo antissentido que atua no processamento do ácido ribonucleico, mensageiro do principal gene modificador da doença, o SMN2, levando a um aumento na produção da proteína SMN. Em ensaios clínicos randomizados, o uso de nusinersena demonstrou melhora da função motora, funcional e redução da necessidade de suporte ventilatório. No Brasil, o medicamento é fornecido pelo Sistema Único de Saúde (SUS), desde o início de 2019, apenas para os pacientes com diagnóstico de AME-5q tipo 1 e que cumprem os critérios de elegibilidade do Protocolo Clínico e Diretrizes Terapêuticas (PCDT) da doença (Brasil, 2019). Apesar de haver discussões na literatura sobre os elevados custos dos medicamentos, não há estudos que avaliaram o real custo por paciente com AME-5q contemplando o consumo de recursos do sistema de saúde e da família, sendo encontrados apenas estudos que avaliaram despesas do sistema de saúde
(Armstrong et al., 2016; Klug et al., 2016) ou contas hospitalares (Darbà \& Marsà, 2019; Liewluck \& Saperstein, 2015).

Acredita-se que os melhores resultados clínicos possam ser obtidos com a combinação dos novos agentes terapêuticos específicos para AME-5q com medidas não farmacológicas (Mercuri et al., 2018). As recomendações na linha de base incluem avaliações de necessidades nutricionais e alimentares, função respiratória, sono, atividades da vida diária e status ortopédico (Arnold et al., 2015; Darras, 2015; Farrar et al., 2018). Por isso, indivíduos com AME-5q são avaliados clinicamente pelo menos a cada seis meses, o que implica custos diretos e indiretos com o manejo da doença.

Compreendem-se como custos diretos os equipamentos e dispositivos relacionados a terapias, dietas especializadas, gastos com adaptação do lar, consultas médicas de rotina e hospitalizações, e como custos indiretos, por exemplo, o planejamento e a readequação que ocorrem nas famílias para atender essas necessidades. Estima-se que os custos indiretos suportados pelas famílias que cuidam de pacientes com AME-5q são altos, mas esses valores não são conhecidos pelo Estado ou sociedade (Farrar et al., 2018; Jeppesen, 2013).

Entende-se que compreender o custo da linha de cuidado de pacientes brasileiros com AME-5q é uma maneira de buscar oportunidades de aprimorar o uso dos recursos de saúde requeridos pelos pacientes. E, dessa forma, identificar formas de melhor distribuir os recursos e subsidiar os cuidados de saúde para a parcela da população portadora da doença. Além disso, considerando os elevados valores relacionados ao manejo farmacológico, estudos econômicos no contexto da AME-5q contribuem também para a literatura científica, além de subsidiarem a gestão de saúde com informações inéditas.

A mensuração de como recursos são consumidos pelos pacientes pode ser feita utilizando-se técnicas de macrocusteio (gross-costing) e microcusteio (micro-costing), sendo as de microcusteio reconhecidas como "padrão-ouro" para valoração de custos, uma vez que permitem analisar os custos dos recursos relativamente ao paciente (Tan et al., 2009; Etges et al., 
2019b, Etges et al., 2020). Por isso, o Ministério da Saúde lançou a Diretriz de Microcusteio como uma forma de padronizar a avaliação de custos para análises econômicas de saúde no Brasil (Etges et al., 2019a); entre os métodos recomendados está o método de custeio baseado em atividades e tempo (TDABC). O TDABC é um método mais preciso para avaliar os custos de cuidados em saúde e, também, para contribuir com as iniciativas de gestão de sistemas de saúde por valor (Etges et al., 2020).

Este estudo teve como objetivo estimar o custo individual ao longo de um ano de acompanhamento de uma coorte brasileira de pacientes diagnosticados com AME-5q, empregando a técnica de microcusteio considerando custos diretos e indiretos.

\section{Métodos}

O estudo caracteriza-se pela análise de uma coorte de pacientes de um centro de referência no tratamento de AME$5 \mathrm{q}$, com coleta retrospectiva de dados para valoração do custo relacionado ao consumo de recursos pelos pacientes. $\mathrm{Na}$ amostra, foram incluídos todos os pacientes com AME$5 q$, provenientes da lista de atendimentos do SUS realizados no ano de 2019, atendidos pelo Serviço de Genética Médica e Unidade de Neurologia Infantil do Hospital de Clínicas de Porto Alegre. Esse estudo econômico foi submetido e aprovado pelo Comitê de Ética em Pesquisa (CAAE: 280806720.7.0000.5327) como um objetivo adicional ao projeto de pesquisa "Criação do Centro de Referência (CR) Estadual para Avaliação, Tratamento e Acompanhamento de Pacientes com Atrofia Muscular Espinhal Tipo 1 (CR AME) no Hospital de Clínicas de Porto Alegre".

Para a medição de custos por paciente, as etapas do TDABC foram seguidas (Keel et al., 2017; Etges et al., 2019b), sendo elas: mapeamento do fluxo de cuidado com as atividades principais às quais o paciente é submetido; identificação de todos os recursos e departamentos que são consumidos pelo paciente; estimativa do gasto total de cada recurso identificado no fluxo de cuidado; estimativa da capacidade horária de cada recurso ou departamento e cálculo da taxa de custo unitário (TCU); análise do tempo utilizado de cada recurso pelo paciente e estruturação das equações de tempo e custos; cálculo do custo por paciente; e análises estatísticas.

\section{Mapeamento do fluxo de cuidado com as atividades principais}

O mapeamento do fluxo de cuidado foi realizado por uma equipe multidisciplinar, incluindo profissionais dos serviços de genética médica, neurologia adulto e infantil, pneumologia, fisiatria e fisioterapia e pesquisadores da área de avaliação de tecnologias em saúde. A sequência de etapas detalhada também considerou os protocolos de cuidado dos pacientes com AME-5q. Os fluxos foram inicialmente desenhados manualmente e, após rodadas de discussão com a equipe, foram documentados, utilizando a plataforma miro.com.

\section{Identificação dos recursos e departamentos consumidos pelo paciente}

Esta etapa foi conduzida a partir da revisão dos registros clínicos em prontuário eletrônico dos pacientes, para identificação das atividades ao longo da rotina de cuidados. A partir dos dados de texto livre registrados pelos profissionais de saúde, foi possível identificar os locais do hospital, equipamentos e profissionais envolvidos nas atividades às quais os pacientes foram submetidos. Para cada atividade, foi identificado o tipo de recurso consumido, sendo esses classificados em: estrutura física da instituição, de profissionais e/ou de uso de equipamentos, exames, medicamentos e materiais. As análises estatísticas foram feitas com a base de dados anonimizados, respeitando a privacidade dos pacientes.

\section{Estimativa do custo total de cada recurso identificado no fluxo de cuidado}

Para cada recurso identificado, foi feito o levantamento de gastos médios anuais com o recurso. Para a estrutura física do hospital, dados dos centros de custos dos departamentos em que o paciente recebeu cuidado foram levantados com a gestão financeira do hospital, sendo considerados custos fixos de depreciação, energia, materiais de apoio, impostos e licenças de sistemas. Para o custo com profissionais, foi utilizada a base salarial do ano de 2019 acrescida de encargos por classe profissional média da instituição.

\section{Estimativa da capacidade horária de cada recurso ou departamento e cálculo da taxa de custo unitário}

Para cada departamento, foi calculada a capacidade de atendimento ao longo de um mês. Para as unidades de internação, o número de leitos disponíveis ao longo de 30 dias por mês, 24 horas por dia, foi considerado. Para os departamentos que estão associados à disponibilidade de sala e equipes, como as salas de ambulatório, foram consideradas as alocações dos profissionais para o cálculo de capacidade. Por exemplo, para o bloco cirúrgico, foram considerados as salas disponíveis por dia e o tempo disponível por dia de cada sala para estimar a capacidade horária de salas cirúrgicas disponíveis ao longo de um mês. Para os profissionais, foram utilizadas as cargas horárias contratadas ao longo de um mês. Com as informações de custo por recursos e capacidade, foi possível calcular as taxas de custo por unidade (TCU) de tempo de cada recurso.

\section{Análise do tempo utilizado de cada recurso pelo paciente e estruturação das equações de tempo e custos}

Para a coleta e a análise de tempos utilizados pelos pacientes em cada atividade, os profissionais envolvidos na linha de cuidado foram questionados sobre o tempo médio de dedicação diária a um paciente com AME-5q. Foram consultados médicos geneticistas, neurologistas adultos e infantis, pneumologistas, pediatras, enfermeiros, psicólogos, fonoaudiólogos e nutricionistas. 
Para estruturar as equações de tempo, a partir da revisão e da análise das evoluções dos pacientes, mapas de consumo dos pacientes foram construídos em planilhas eletrônicas. Para cada paciente, foi construído um mapa, com o cruzamento de informações: "o que e quando aconteceu, por quem foi feito e tempo dedicado". Essas tabelas individuais representam a principal fonte de dados para os cálculos de custos por atividade e recursos por paciente e foram construídas para as análises do custo anual de paciente com AME-5q.

\section{Cálculo do custo por paciente}

Para avaliar o custo individual por paciente, multiplicou-se o tempo utilizado de cada recurso pela TCU de tempo e, na sequência, foram somados os custos diretos identificados em cada atividade, como medicamentos, materiais, exames dentro e fora do hospital, considerando o custo de aquisição do hospital (não incluindo qualquer margem de lucro). Para o custo do nusinersena, foi considerado o valor de R\$159.000 por frasco fornecido pela Secretaria Estadual de Saúde do Estado do Rio Grande do Sul.

Além disso, foi estimado o custo com transporte para os deslocamentos com as consultas e internações hospitalares. Para os pacientes que residem fora de Porto Alegre, o cálculo de custo com transporte considerou a quilometragem entre o município e a cidade de Porto Alegre, um parâmetro de custo de R\$ 1,20 por quilômetro rodado e a necessidade da realização de duas viagens (ida e volta). Para os pacientes que residem em Porto Alegre, foi considerado o custo de uma passagem urbana do município de Porto Alegre de $R \$ 4,55$ para o paciente e um acompanhante.

\section{Estimativa do gasto familiar com o manejo da doença}

Entendendo que o espectro da doença AME-5q é crônico e acarreta custos e despesas aos familiares, foram estimados os custos anuais que os familiares e pacientes tiveram no decorrer do ano de 2019. Para isso, entrevistas telefônicas com os familiares foram conduzidas com perguntas que objetivam estimar o gasto mensal relacionado à doença pela família do paciente (Anexo I).

Para a análise do custo de oportunidade relacionado à parada com as atividades profissionais de algum dos familiares, foi considerada a última referência salarial do familiar para estimar a redução de renda anual da família por causa da doença.

\section{Análise dos dados}

Com a informação de custo calculada por atividade e recursos consumidos, foram conduzidas análises descritivas da informação de custos por paciente estratificadas em: custo anual hospitalar e ambulatorial por paciente com uso ou não do nusinersena; custo hospitalar anual por paciente por tipo de AME-5q; custo hospitalar anual por paciente por faixa etária (0-<2; 2-<6; 6-11; 12-17; 18-25; mais de 26 anos); custo anual por paciente com transporte para consultas e hospitalizações; custo anual com cuidados domiciliares ou terapia de suporte em casa; e, por fim, custo anual por paciente com os cuidados da doença estratificado em custos hospitalares, de transporte e da família. Para essa última, a divisão de custos buscou respeitar a perspectiva da informação de custos e origem dos recursos. Os custos hospitalares nesse caso receberam fomento público do município de Porto Alegre, Ministérios da Saúde e da Educação; quando o paciente é residente no município de Porto Alegre, os municípios de origem de cada paciente ou a família arcam com os custos com transporte, e as próprias famílias arcam com os custos domiciliares.

Todas as informações de custos foram coletadas e estão expressas em moeda brasileira (reais) no ano de 2019.

\section{Resultados}

Foram analisados os custos de 43 pacientes que registraram algum tratamento para AME-5q no HCPA no ano de 2019. A Tabela 1 apresenta a caracterização clínica da amostra estudada.

\section{Mapeamento do fluxo de cuidado com as atividades principais às quais o paciente é submetido}

O mapeamento do fluxo de cuidado hospitalar ou ambulatorial buscou representar as possibilidades de manejo de cuidado adotadas com os pacientes com AME- $5 q$ desde a chegada à instituição até a alta deles. A Figura 1 apresenta o macrofluxo de cuidados desses pacientes.

A partir do detalhamento do fluxo dos pacientes, para cada um dos recursos de profissionais e estrutura física da instituição utilizados ao longo do tratamento dos pacientes, foi calculada a TCU em reais por hora $(R \$ / h)$, respeitando as características financeiras da instituição estudada. A Tabela 2 apresenta os valores de TCU de cada um dos recursos identificados.

\section{Tempo de dedicação dos profissionais no cuidado dos pacientes com AME-5q e estruturação das equações de tempo e custos}

Para as atividades de cuidado dos pacientes, foi estimado o tempo médio de envolvimento dos profissionais de saúde a partir do relato e validação dos próprios profissionais. Essa informação foi utilizada para estimar o custo para cada um dos pacientes ao ser cruzada com o número de vezes que foi realizada alguma atividade de cada profissional com os pacientes. O Anexo II apresenta o tempo unitário de atividades de rotina dos profissionais com cuidados assistenciais dedicados aos pacientes com AME-5q. Para o custo com estrutura física, o tempo que cada paciente ficou em cada uma das unidades foi multiplicado pelas respectivas TCUs de cada unidade. Para o custo com consumo de profissionais de saúde, o tempo de dedicação dos profissionais ao longo das internações dos pacientes foi multiplicado pela TCU de cada um dos profissionais. 
Tabela 1. Caracterização da amostra

\begin{tabular}{|c|c|c|c|c|}
\hline & $\mathrm{N}$ & $\begin{array}{l}\text { Pacientes com } \\
\text { internações } \\
\text { hospitalares }\end{array}$ & $\begin{array}{l}\text { Pacientes sem } \\
\text { internações } \\
\text { hospitalares } \\
\text { (apenas } \\
\text { ambulatoriais) }\end{array}$ & $\begin{array}{c}\text { Pacientes } \\
\text { em uso de } \\
\text { nusinersena* }\end{array}$ \\
\hline Total de pacientes & $43(100 \%)$ & $9(21 \%)$ & 34 (79\%) & $5(12 \%)$ \\
\hline \multicolumn{5}{|c|}{ Classificação da doença } \\
\hline AME 1 & $11(25 \%)$ & $5(12 \%)$ & $6(14 \%)$ & $3(7 \%)$ \\
\hline AME 2 & $15(35 \%)$ & $3(7 \%)$ & $12(28 \%)$ & $2(5 \%)$ \\
\hline AME 3 & $15(35 \%)$ & $1(2 \%)$ & $14(32 \%)$ & - \\
\hline AME 4 & $2(5 \%)$ & - & $2(5 \%)$ & - \\
\hline \multicolumn{5}{|l|}{ Faixa etária } \\
\hline $0-<2$ anos & $6(14 \%)$ & $3(7 \%)$ & $3(7 \%)$ & $3(7 \%)$ \\
\hline $2-<6$ anos & $6(14 \%)$ & $3(7 \%)$ & $3(7 \%)$ & $1(2 \%)$ \\
\hline 6-11 anos & $4(9 \%)$ & $1(2 \%)$ & $3(7 \%)$ & $1(2 \%)$ \\
\hline 12-17 anos & $4(9 \%)$ & - & $4(9 \%)$ & - \\
\hline 18-25 anos & $9(21 \%)$ & $2(5 \%)$ & $7(16 \%)$ & - \\
\hline + de 26 anos & $14(33 \%)$ & - & $14(33 \%)$ & - \\
\hline
\end{tabular}

* Em 2019.

\section{Custos hospitalares e ambulatoriais}

O custo médio dos 38 pacientes que não fizeram uso do nusinersena foi de R\$ 4.870 (mín. R\$ 190 - máx. R\$ 67.138), enquanto o custo médio dos cinco pacientes que fizeram uso do medicamento foi de R\$ 600.347 (mín. R\$ 342.679 máx. R\$ 813.513), e neste último grupo o medicamento representou $91 \%$ do valor. Para esses pacientes, o custo com o procedimento intratecal para o uso do medicamento foi em média de R\$ 2.046 para pacientes AME tipo 1 e R\$ 3.175 para pacientes AME tipo 2. Para os demais fenótipos da doença, não foi possível identificar na amostra estudada pacientes que utilizaram o medicamento. Ao ser avaliada a composição de custos hospitalares para o grupo de pacientes que não receberam o nusinersena, o custo com profissionais médicos e equipe multidisciplinar de cuidado excede $50 \%$ do custo, independentemente do tipo de AME-5q. A Figura 2 apresenta a composição dos custos por tipo de AME-5q para esse grupo de pacientes. No que diz respeito às consultas ambulatoriais, os pacientes com AME tipo 1 foram os que realizaram mais consultas, em média seis ao ano; os pacientes com AME tipo 2 realizaram quatro consultas, os com AME tipo 3, três consultas e os com AME tipo 4, quatro consultas. A amostra de pacientes com AME-5q tipo 4 ficou restrita às consultas ambulatoriais, por isso não teve influência dos custos de exames e medicamentos.

Também foi possível observar que os pacientes mais jovens apresentaram maior custo hospitalar com o tratamento da doença no grupo de pacientes que não utilizaram nusinersena, com custo médio de R\$23.254 entre os pacientes menores de 2 anos de idade e demais resultados apresentados na Tabela 3.

Para o grupo de pacientes que utilizaram o medicamento nusinersena, três pacientes pertencem à faixa etária de 0-2 anos, um paciente, à faixa etária de 2-6 anos e um paciente, à faixa etária entre 6-11 anos. Os custos médios por faixa etária foram, respectivamente, de R\$ 514.937 (mín. R\$318.074 - máx. R\$ 802.566), R\$ 646.550 e R\$ 806.069. O fator que contribuiu para as diferenças de custos observadas foi o período de acompanhamento dos pacientes. Os pacientes seguem um mesmo ciclo de uso do medicamento, mas, ao longo do ano de acompanhamento, alguns receberam mais doses do que outros por terem iniciado o tratamento em datas diferentes.

\section{Custos com transporte}

No que diz respeito ao transporte, foi observado um custo anual médio de R\$ 4.895 (mín. R\$ 18 - máx. R\$ 73.382). Os pacientes com AME-5q tipo 1 e os pacientes mais jovens (0-2 anos) foram os que registraram maior custo médio com transporte, respectivamente, de R\$ 10.129 (mín. R\$ 54 - máx. R\$ 73.382) e R\$ 16.085 (mín. R\$ 293 - máx. R\$ 73). O Anexo III apresenta a informação detalhada do custo com transporte. Para a maioria dos pacientes, esse custo é subsidiado pelos municípios, exceto para os cinco pacientes de Porto Alegre (P10, P12, P16, P36 e P37), para os quais se considerou a referência passagem de ônibus urbana, aferindo-se um custo médio de R\$ 200 por ano. 


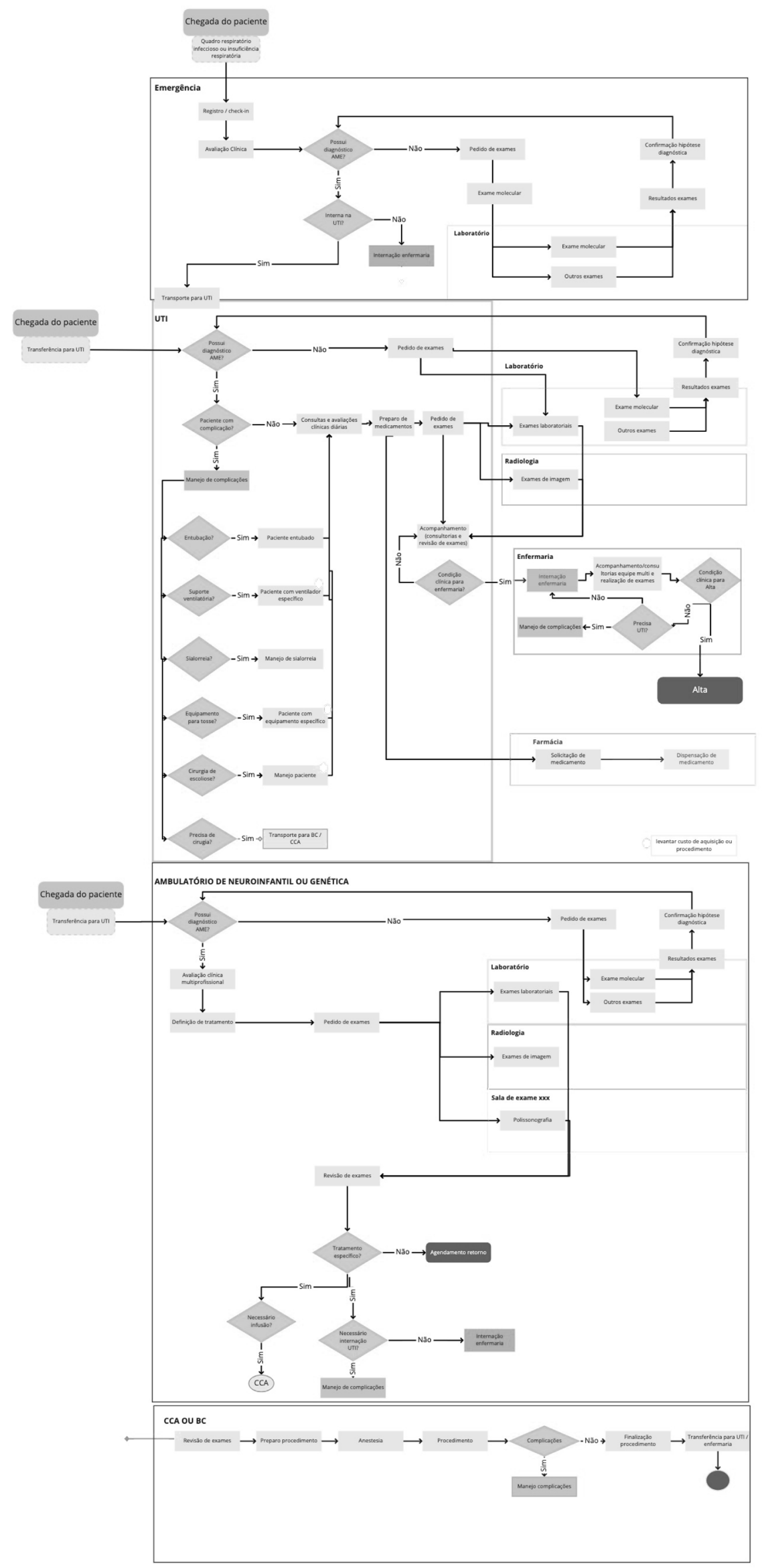

Figura 1. Macrofluxo de cuidados de pacientes. 
Tabela 2. Taxas de custo por unidade de tempo

\begin{tabular}{|c|c|}
\hline Profissionais & TCU Profissionais (R\$/h)* \\
\hline Médico & $\mathrm{R} \$ 113$ \\
\hline Enfermeiro & $R \$ 59$ \\
\hline Técnico de Enfermagem & $R \$ 35$ \\
\hline Psicólogo & $R \$ 56$ \\
\hline Nutricionista & $\mathrm{R} \$ 58$ \\
\hline Fisioterapeuta & $\mathrm{R} \$ 59$ \\
\hline Fonoaudiólogo & $\mathrm{R} \$ 58$ \\
\hline Técnico de Radiologia & $\mathrm{R} \$ 51$ \\
\hline Farmacêutico & $\mathrm{R} \$ 71$ \\
\hline Professores/preceptores & $\mathrm{R} \$ 154$ \\
\hline Residente médico & $\mathrm{R} \$ 18$ \\
\hline Estrutura Física & TCU Estruturas hospitalares $(\mathrm{R} \$ / \mathrm{h})^{* *}$ \\
\hline Emergência & $R \$ 5$ \\
\hline Internação pediátrica & $R \$ 6$ \\
\hline Centro de terapia intensiva & $R \$ 39$ \\
\hline Ambulatório & $\mathrm{R} \$ 9$ \\
\hline Centro cirúrgico & $\mathrm{R} \$ 248$ \\
\hline Sala de recuperação & $\mathrm{R} \$ 14$ \\
\hline Farmácia & $\mathrm{R} \$ 31$ \\
\hline
\end{tabular}

* Foi calculada considerando salários e encargos por classe de profissionais.

** Não contempla custos com profissionais de saúde, materiais e medicamentos. Inclui custos alocados ao departamento, por exemplo, mas não restritos a depreciação, energia, licenças e materiais de apoio.

Composição do custo anual por tipo de AME

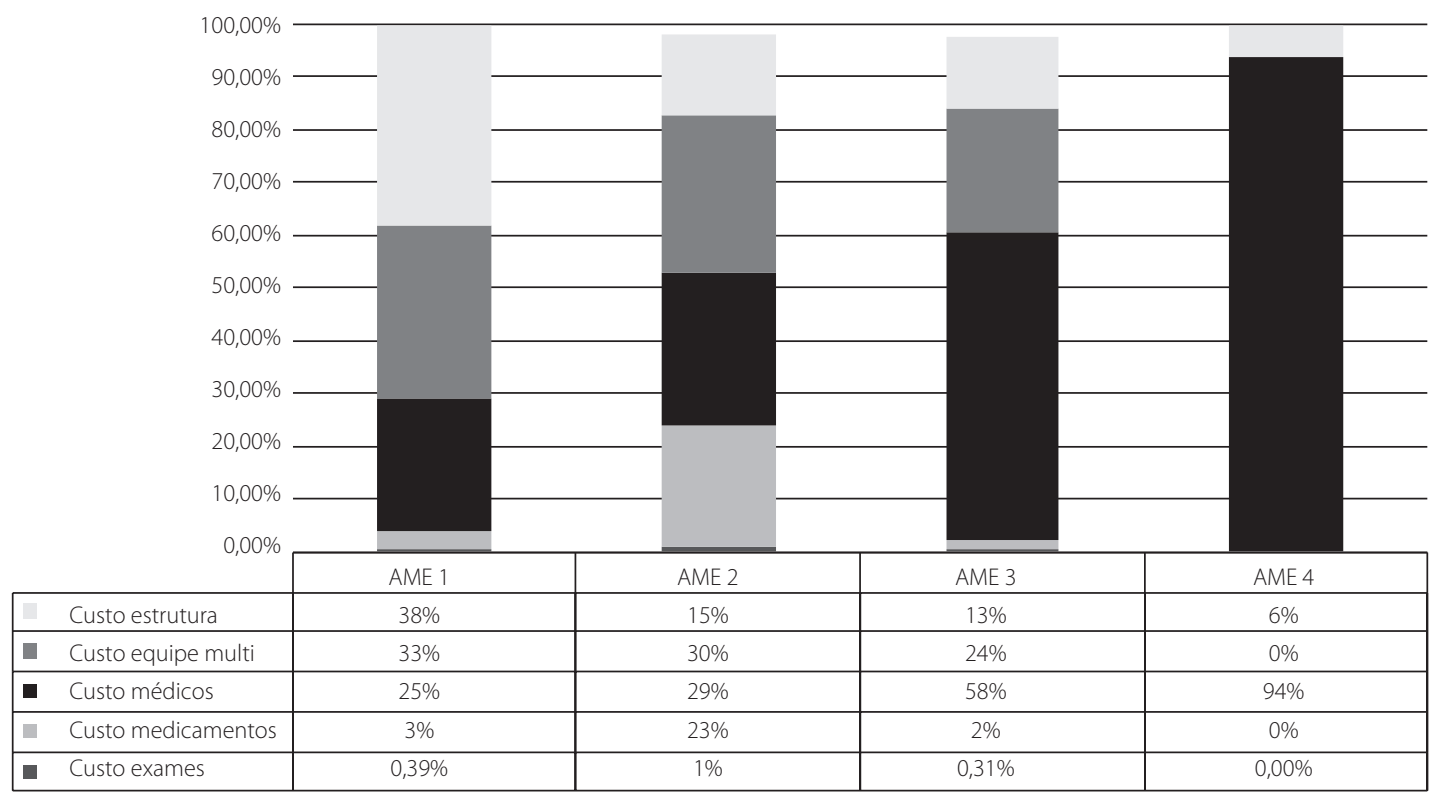

Figura 2. Composição de custos para pacientes que não fizeram uso do medicamento nusinersena.

Os medicamentos representados por classes terapêuticas de analgésicos, antitérmicos, laxantes osmóticos, anticolinérgicos, expectorantes mucolíticos, corticosteroides e antiepiléticos representam aproximadamente $80 \%$ do uso total de medicamentos durante a internação. Os medicamentos mais caros, porém pouco frequentes, são da classe de imunossupressores, antibióticos (cefalosporinas de segunda geração, penicilinas) e vitamina D. Materiais dizem respeito a nota de sala de procedimentos realizados durante a internação. Exames contêm os exames bioquímicos de rotina e de imagem. A tabela com os valores em reais da composição de custos por tipo de AME está no Anexo V. 
Tabela 3. Custo hospitalar por faixa etária

\begin{tabular}{|c|c|c|c|c|c|c|}
\hline Faixa de idade & $\mathrm{N}$ & Custo ambulatorial & Custo de internação & Custo de exames & $\begin{array}{l}\text { Custo de materiais e } \\
\text { medicamentos }\end{array}$ & Custo anual \\
\hline $0-<2$ anos & 3 & $\begin{array}{c}\text { Média } R \$ 2.982 \\
(1.103-6.323) \\
\text { Med R\$1.521 }\end{array}$ & $\begin{array}{c}\text { Média R\$19.229 } \\
\text { (0-57.689) } \\
\text { Med R\$ } 0\end{array}$ & $\begin{array}{c}\text { Média } R \$ 110 \\
(0-330) \\
\text { Med } R \$ 0\end{array}$ & $\begin{array}{c}\text { Média R\$ } 931 \\
(0-2.794) \\
\text { Med } R \$ 0\end{array}$ & $\begin{array}{c}\text { Média } R \$ 23.254 \\
(1.103-67.138) \\
\text { Med } R \$ 1.521\end{array}$ \\
\hline $2-<6$ anos & 5 & $\begin{array}{c}\text { Média R\$ } 915 \\
(437-1.267) \\
\text { Med } R \$ 943\end{array}$ & $\begin{array}{c}\text { Média } R \$ 1.938 \text { (0- } \\
7.576) \\
\text { Med R\$ } 0\end{array}$ & - & $\begin{array}{c}\text { Média R\$ } 13 \text { (0-66) } \\
\text { Med R\$ } 0\end{array}$ & $\begin{array}{c}\text { Média } R \$ 2.867 \\
(437-8.910) \\
\text { Med } R \$ 943\end{array}$ \\
\hline 6-11 anos & 3 & $\begin{array}{c}\text { Média R\$1.321 } \\
(945-1.525) \\
\text { Med R\$1.493 }\end{array}$ & $\begin{array}{c}\text { Média } R \$ 82,39 \\
(0-247) \\
\text { Med R\$ } 0\end{array}$ & - & - & $\begin{array}{c}\text { Média R\$1.403 } \\
(945-1.772) \\
\text { Med R\$1.493 }\end{array}$ \\
\hline 12-17 anos & 4 & $\begin{array}{c}\text { Média R\$ } 826 \\
(190-1.751) \\
\text { Med R\$ } 682\end{array}$ & - & - & - & $\begin{array}{c}\text { Média R\$ } 826 \\
(190-1.751) \\
\text { Med } R \$ 682\end{array}$ \\
\hline 18-25 anos & 9 & $\begin{array}{c}\text { Média } \mathrm{R} \$ 1.501 \\
(304-5.842) \\
\text { Med } R \$ 1.146\end{array}$ & $\begin{array}{c}\text { Média } R \$ 5.313 \\
(0-40.119) \\
\text { Med R\$ } 0\end{array}$ & $\begin{array}{c}\text { Média } R \$ 103 \\
(0-866) \\
\text { Med } R \$ 0\end{array}$ & $\begin{array}{c}\text { Média R\$ } 2.049 \\
(0-18.026) \\
\text { Med R\$ } 0 \\
\end{array}$ & $\begin{array}{c}\text { Média } R \$ 8.968 \\
(304-64.854) \\
\text { Med R\$ } 1.146 \\
\end{array}$ \\
\hline + de 26 anos & 14 & $\begin{array}{c}\text { Média } R \$ 909 \\
(304-2.296) \\
\text { Med } R \$ 794\end{array}$ & - & - & - & $\begin{array}{c}\text { Média R\$909 } \\
\text { (304-2.296) } \\
\text { Med R\$ } 794\end{array}$ \\
\hline
\end{tabular}

Dados expressos em média (mínimo e máximo) e mediana (med). Os medicamentos representados por classes terapêuticas de analgésicos, antitérmicos, laxantes osmóticos, anticolinérgicos, expectorantes mucolíticos, corticosteroides e antiepiléticos representam aproximadamente 80\% do uso total de medicamentos durante a internação. Os medicamentos mais caros, porém pouco frequentes, são da classe de imunossupressores, antibióticos (cefalosporinas de segunda geração, penicilinas) e vitamina D. Materiais dizem respeito a nota de sala de procedimentos realizados durante a internação.

Quando considerado o transporte ao custo hospitalar dos pacientes, o custo com transporte tem representatividade de $52 \%$ do custo anual hospitalar e ambulatorial (excluindo o gasto familiar adicional) para pacientes que não fizeram uso de nusinersena ( $n=38)$; para os pacientes que fizeram uso do medicamento $(n=5)$, tendo em vista o alto custo deste, o custo com transporte representa em torno de $1 \%$.

\section{Custo anual com cuidados domiciliares ou terapia de suporte familiar}

Os familiares de 30 pacientes foram entrevistados (Anexo l), e o gasto médio anual familiar por paciente reportado foi de R\$ 32.262 (mín. R\$ 240 - máx. R\$ 110.952). Essa informação contempla gastos com consultas particulares e convênios (média anual de R\$ 10.763 - 33\%), medicamentos (média anual de R\$ 1.600 - 5\%), cuidadores (média anual de R\$ 2.629 - 8\%) e outros (média anual de R\$ 3.511 - 11\%) e a necessidade de os familiares terem deixado de trabalhar por causa dos cuidados necessários pelo portador da doença (média anual de $R \$ 14.232$ - 43\%). Para esse último, reitera-se que essa informação contempla apenas a renda que deixou de ser gerada com base no último salário que o familiar registrou antes de parar suas atividades, deixando-se de fora demais custos indiretos e sociais relacionados. Para o item outros, o relato mais frequentemente recebido foi o gasto com fraldas e alimentação especializada.

Ao ser estratificada a informação de gasto anual da família com os cuidados da doença, foi observado um gasto maior com os pacientes com AME-5q tipo 1 (média de R\$ 34.298, mín. R\$ 0 - máx. R\$ 163.480) e no grupo etário mais jovem, respectivamente, nas faixas de idade 2-6 anos (média de R\$ 46.821) seguidas da faixa de idade $0-2$ anos (média de R\$ 41.818). A Tabela 4 apresenta os gastos médios por tipo de AME-5q e por idade e indica que os gastos mais representativos são em relação ao familiar que deixou de trabalhar e às consultas particulares.

\section{Custo anual total com os cuidados de pacientes com AME-5q}

Considerando-se os custos hospitalares e ambulatoriais, de transporte e de cuidados domiciliares, foi consolidada a estimativa do custo médio anual por paciente com AME-5q por fenótipo da doença e faixa etária do paciente (Anexo IV), independentemente do manejo de tratamento. Pacientes com AME-5q tipos 1 e 2 e pacientes mais jovens apresentaram o maior custo total com os cuidados da doença, sendo a maioria justificada por custos hospitalares e ambulatoriais, seguidos dos gastos com cuidados domiciliares.

Em relação ao tipo de fenótipo da doença, os pacientes com AME-5q tipos 1 e 2 apresentam maior custo anual total, especialmente porque fazem parte dessa população os pacientes em uso de nusinersena e os pacientes que registraram mais consultas e internações, fazendo com que o custo anual hospitalar (sujeito ao uso de nusinersena) represente $72 \%$ e $75 \%$, respectivamente, do custo total de pacientes com AME-5q tipos 1 e 2 (Figura 3). 
Tabela 4. Composição do gasto familiar por ano por tipo de AME-5q e por faixa de idade

\begin{tabular}{|c|c|c|c|c|c|c|c|c|}
\hline $\begin{array}{l}\text { Tipo/Faixa } \\
\text { etária }\end{array}$ & $\mathrm{N}$ & Medicamentos & $\begin{array}{l}\text { Consultas com } \\
\text { convênio }\end{array}$ & $\begin{array}{l}\text { Consultas } \\
\text { particulares }\end{array}$ & $\begin{array}{c}\text { Familiar que } \\
\text { deixou de } \\
\text { trabalhar }\end{array}$ & Cuidador & Outros & Custo anual \\
\hline AME 1 & $1137 \%$ & $\begin{array}{c}\text { Média } R \$ 567 \\
(0-3.600) \\
\text { Med } R \$ 0\end{array}$ & $\begin{array}{c}\text { Média R\$ } \\
1.090 \\
(0-5.880) \\
\text { Med R\$0 }\end{array}$ & $\begin{array}{c}\text { Média } \\
\mathrm{R} \$ 6.452 \\
(0-40.000) \\
\text { Med } \mathrm{R} \$ 0\end{array}$ & $\begin{array}{c}\text { Média } \\
\text { R\$19.338 } \\
(0-60.000) \\
\text { Med } R \$ 16.200\end{array}$ & $\begin{array}{c}\text { Média } \\
\text { R\$ 1.636 } \\
\text { (0-18.000) } \\
\text { Med R\$0 }\end{array}$ & $\begin{array}{c}\text { Média } \\
\text { R\$5.215 } \\
(0-36.000) \\
\text { Med R\$0 }\end{array}$ & $\begin{array}{c}\text { Média } \\
R \$ 34.298 \\
(0-163.480) \\
\text { Med } R \$ 18.000\end{array}$ \\
\hline AME 2 & $1137 \%$ & $\begin{array}{c}\text { Média } \\
\mathrm{R} \$ 2.956 \\
(0-14.400) \\
\text { Med } \mathrm{R} \$ 600\end{array}$ & $\begin{array}{c}\text { Média } \\
\text { R\$ 1.974 } \\
(0-7.200) \\
\text { Med } \mathrm{R} \$ 1.800\end{array}$ & $\begin{array}{c}\text { Média } \\
\text { R\$ 13.525 } \\
(0-86.400) \\
\text { Med R\$ } 1.200\end{array}$ & $\begin{array}{c}\text { Média } \\
\text { R\$ 19.338 } \\
(0-60.000) \\
\text { Med R\$11.976 }\end{array}$ & $\begin{array}{c}\text { Média } \\
\text { R\$1.636 } \\
(0-18.000) \\
\text { Med } \mathrm{R} \$ 1.500\end{array}$ & $\begin{array}{c}\text { Média } \\
\text { R\$2.005 } \\
(0-11.976) \\
\text { Med R\$0 }\end{array}$ & $\begin{array}{c}\text { Média } \\
\text { R\$ 42.261 } \\
\text { (0-193.176) } \\
\text { Med R\$ 8.004 }\end{array}$ \\
\hline AME 3 & $\begin{array}{c}7 \\
7 \%\end{array}$ & $\begin{array}{c}\text { Média R\$ } 463 \\
(0-1.800) \\
\text { Med R\$ } 6.000 \\
\text { DP } 611\end{array}$ & $\begin{array}{c}\text { Média } \\
\text { R\$3.012 } \\
(0-13.800) \\
\text { Med } \mathrm{R} \$ 504\end{array}$ & $\begin{array}{c}\text { Média } \\
R \$ 5.709 \\
(0-11.040) \\
\text { Med } \mathrm{R} \$ 6.000\end{array}$ & $\begin{array}{c}\text { Média } \\
\text { R\$1.711 } \\
(0-11.976) \\
\text { Med R\$0 }\end{array}$ & $\begin{array}{c}\text { Média } \\
\text { R\$ } 1500 \\
(0-6.000) \\
\text { Med R\$0 }\end{array}$ & $\begin{array}{c}\text { Média } \\
\mathrm{R} \$ 3.703 \\
(0-25.080) \\
\text { Med } \mathrm{R} \$ 0\end{array}$ & $\begin{array}{c}\text { Média } \\
R \$ 16.098 \\
(0-69.696) \\
\text { Med } R \$ 32.364\end{array}$ \\
\hline AME 4 & $\begin{array}{c}1 \\
3 \%\end{array}$ & $\begin{array}{c}\text { Média } \\
R \$ 6.000 \\
\text { Med } R \$ 6.000)\end{array}$ & - & $\begin{array}{c}\text { Média } \\
R \$ 8.400 \\
\text { Med } R \$ 8.400\end{array}$ & $\begin{array}{l}\text { Média } 17.964 \\
\text { Med R\$17.964 }\end{array}$ & - & - & $\begin{array}{c}\text { Média } \\
R \$ 32.364 \\
\text { Med } R \$ 32.364\end{array}$ \\
\hline \multicolumn{9}{|c|}{ Cuidados domiciliares por faixa etária } \\
\hline $0-2$ anos & $\begin{array}{c}6 \\
20 \%\end{array}$ & $\begin{array}{c}\text { Média } R \$ 440 \\
(0-1.200) \\
\text { Med } R \$ 120\end{array}$ & $\begin{array}{c}\text { Média } \\
\mathrm{R} \$ 1.232 \\
(0-5.880) \\
\text { Med } \mathrm{R} \$ 0\end{array}$ & $\begin{array}{c}\text { Média } \\
\text { R\$ 11.761 } \\
(0-40.000) \\
\text { Med } \mathrm{R} \$ 285\end{array}$ & $\begin{array}{c}\text { Média } \\
\text { R\$ 12.996 } \\
(0-26.004) \\
\text { Med } R \$ 11.876\end{array}$ & - & $\begin{array}{c}\text { Média } \\
R \$ 8.960 \\
(0-36.000) \\
\text { Med } \mathrm{R} \$ 2.525\end{array}$ & $\begin{array}{c}\text { Média } \\
\text { R\$35.389 } \\
(0-109.084) \\
\text { Med R\$ 14.901 }\end{array}$ \\
\hline 2-6 anos & $\begin{array}{c}5 \\
17 \%\end{array}$ & $\begin{array}{c}\text { Média } R \$ 480 \\
(0-2.400) \\
\text { Med } R \$ 0\end{array}$ & $\begin{array}{c}\text { Média } \\
\text { R\$ 1.375 } \\
(0-3.516) \\
\text { Med R\$0 }\end{array}$ & $\begin{array}{c}\text { Média } R \$ 320 \\
(0-1.200) \\
\text { Med } R \$ 0\end{array}$ & $\begin{array}{c}\text { Média } \\
\text { R\$24.955 } \\
(11.976- \\
60.000) \\
\text { Med R\$16.800 }\end{array}$ & $\begin{array}{c}\text { Média } \\
\mathrm{R} \$ 3.600 \\
(0-18.000) \\
\text { Med } \mathrm{R} \$ 0\end{array}$ & $\begin{array}{c}\text { Média } R \$ 504 \\
(0-1.320) \\
\text { Med } R \$ 0\end{array}$ & $\begin{array}{c}\text { Média } \\
\text { R\$31.234 } \\
(11.976- \\
\text { 86.436) } \\
\text { Med } R \$ 16.800\end{array}$ \\
\hline $6-11$ anos & $\begin{array}{c}4 \\
13 \%\end{array}$ & $\begin{array}{l}\text { Média } R \$ 1.500 \\
\quad(0-3.600) \\
\text { Med R\$ } 1.200\end{array}$ & $\begin{array}{c}\text { Média } \\
\text { R\$3.030 } \\
(0-7.200) \\
\text { Med } \mathrm{R} \$ 0\end{array}$ & $\begin{array}{c}\text { Média } \\
\text { R\$ 12.275 } \\
(0-43.700) \\
\text { Med } \mathrm{R} \$ 2.700\end{array}$ & $\begin{array}{c}\text { Média } \\
R \$ 30.300 \\
(15.600- \\
54.000) \\
\text { Med } R \$ 25.800\end{array}$ & $\begin{array}{c}\text { Média } \\
R \$ 4.800 \\
(0-13.200) \\
\text { Med } \mathrm{R} \$ 3.000\end{array}$ & $\begin{array}{c}\text { Média } R \$ 600 \\
(0-2.400) \\
\text { Med } R \$ 0\end{array}$ & $\begin{array}{c}\text { Média } \\
\text { R\$52.505 } \\
(15.600- \\
124.100) \\
\text { Med } R \$ 35.160\end{array}$ \\
\hline 12-17 anos & $\begin{array}{c}1 \\
3 \%\end{array}$ & $\begin{array}{c}\text { Média } \\
\text { R\$ } 12.000 \\
(12.000- \\
12.000) \\
\text { Med } R \$ 12.000\end{array}$ & $\begin{array}{c}\text { Média } \\
\text { R\$ } 2.570 \\
(2.570-7.200) \\
\text { Med } \mathrm{R} \$ 2.570\end{array}$ & - & - & $\begin{array}{c}\text { Média } \\
\text { R\$ 19.200 } \\
(0-19.200) \\
\text { Med } R \$ 19.200\end{array}$ & - & $\begin{array}{c}\text { Média } \\
R \$ 33.770 \\
(33.700- \\
124.100) \\
\text { Med } R \$ 33.770\end{array}$ \\
\hline $18-25$ anos & $\begin{array}{c}6 \\
20 \%\end{array}$ & $\begin{array}{c}\text { Média } R \$ 220 \\
(0-720) \\
\text { Med } R \$ 0\end{array}$ & $\begin{array}{c}\text { Média } R \$ 514 \\
\text { (0-1.800) } \\
\text { Med } R \$ 0\end{array}$ & $\begin{array}{c}\text { Média } \\
\mathrm{R} \$ 16.240 \\
(0-86.400) \\
\text { Med R\$0 }\end{array}$ & $\begin{array}{c}R \$ 3.992 \\
(0-23.952) \\
\text { Med } R \$ 0\end{array}$ & $\begin{array}{c}\text { Média } \\
\text { R\$ - } \\
\text { Med R\$ } 0\end{array}$ & $\begin{array}{c}\text { Média } \\
\text { R\$4.356 } \\
(0-25.080) \\
\text { Med R\$50 }\end{array}$ & $\begin{array}{c}\text { Média } \\
\text { R\$ 25.322 } \\
\text { (0-137.952) } \\
\text { Med R\$440 }\end{array}$ \\
\hline $\begin{array}{l}+ \text { de } 26 \\
\text { anos }\end{array}$ & $\begin{array}{c}8 \\
27 \%\end{array}$ & $\begin{array}{c}\text { Média } \\
\text { R\$2.955 } \\
(0-14.400) \\
\text { Med R\$ } 0\end{array}$ & $\begin{array}{c}\text { Média } \\
\mathrm{R} \$ 2.842 \\
(0-13.800) \\
\text { Med } \mathrm{R} \$ 0\end{array}$ & $\begin{array}{c}\text { Média } \\
R \$ 6.175 \\
(2.000-10.080) \\
\text { Med } \mathrm{R} \$ 6.600\end{array}$ & $\begin{array}{c}R \$ 9.730 \\
(0-47.904) \\
\text { Med } R \$\end{array}$ & $\begin{array}{c}\text { Média } \\
R \$ 2.809 \\
(0-11.976) \\
\text { Med } R \$ 1.500\end{array}$ & $\begin{array}{c}\text { Média } \\
\mathrm{R} \$ 2.564 \\
(0-11.976) \\
\text { Med } \mathrm{R} \$ 0\end{array}$ & $\begin{array}{c}\text { Média } \\
\text { R\$27.077 } \\
(2.000- \\
110.136) \\
\text { Med } \mathrm{R} \$ 9.810\end{array}$ \\
\hline
\end{tabular}

Em relação à faixa etária, pacientes mais jovens apresentaram um custo total maior do que os demais (Figura 4), também justificado pelo maior consumo de recursos hospitalares e por concentrarem os pacientes que fizeram uso de nusinersena (três pacientes menores de 2 anos de idade, um paciente com menos de 6 anos de idade e um com menos de 11 anos de idade). 
$R \$ 250.000,00$

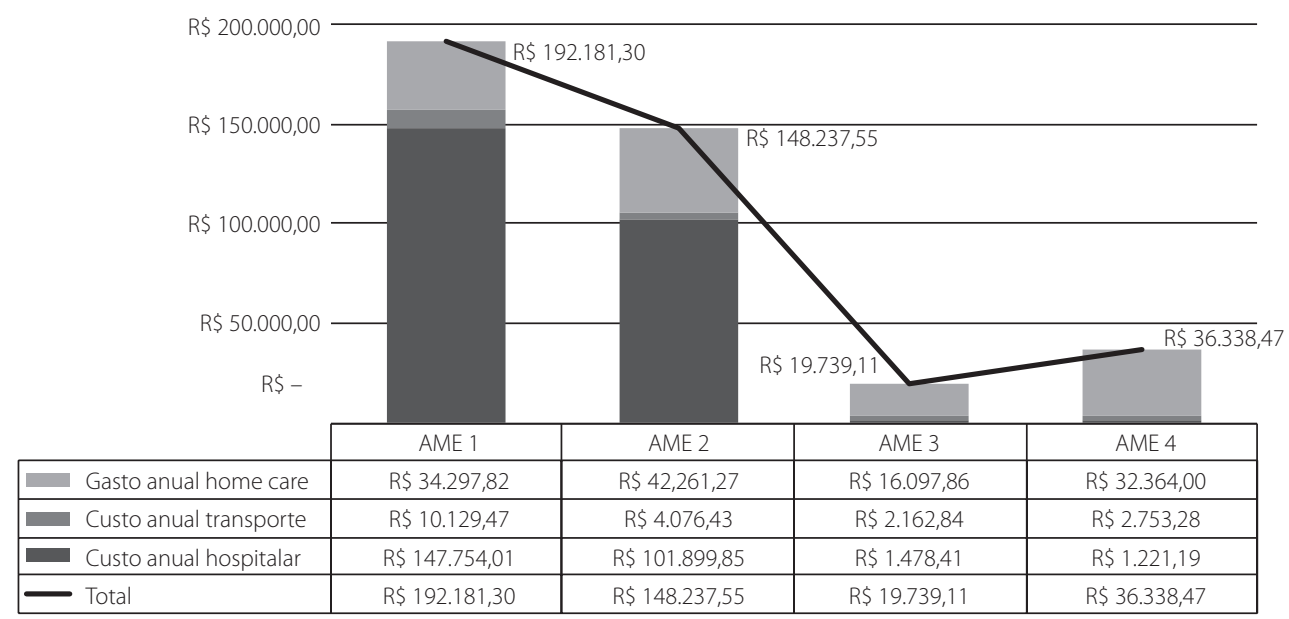

Figura 3. Custo total por tipo de paciente.

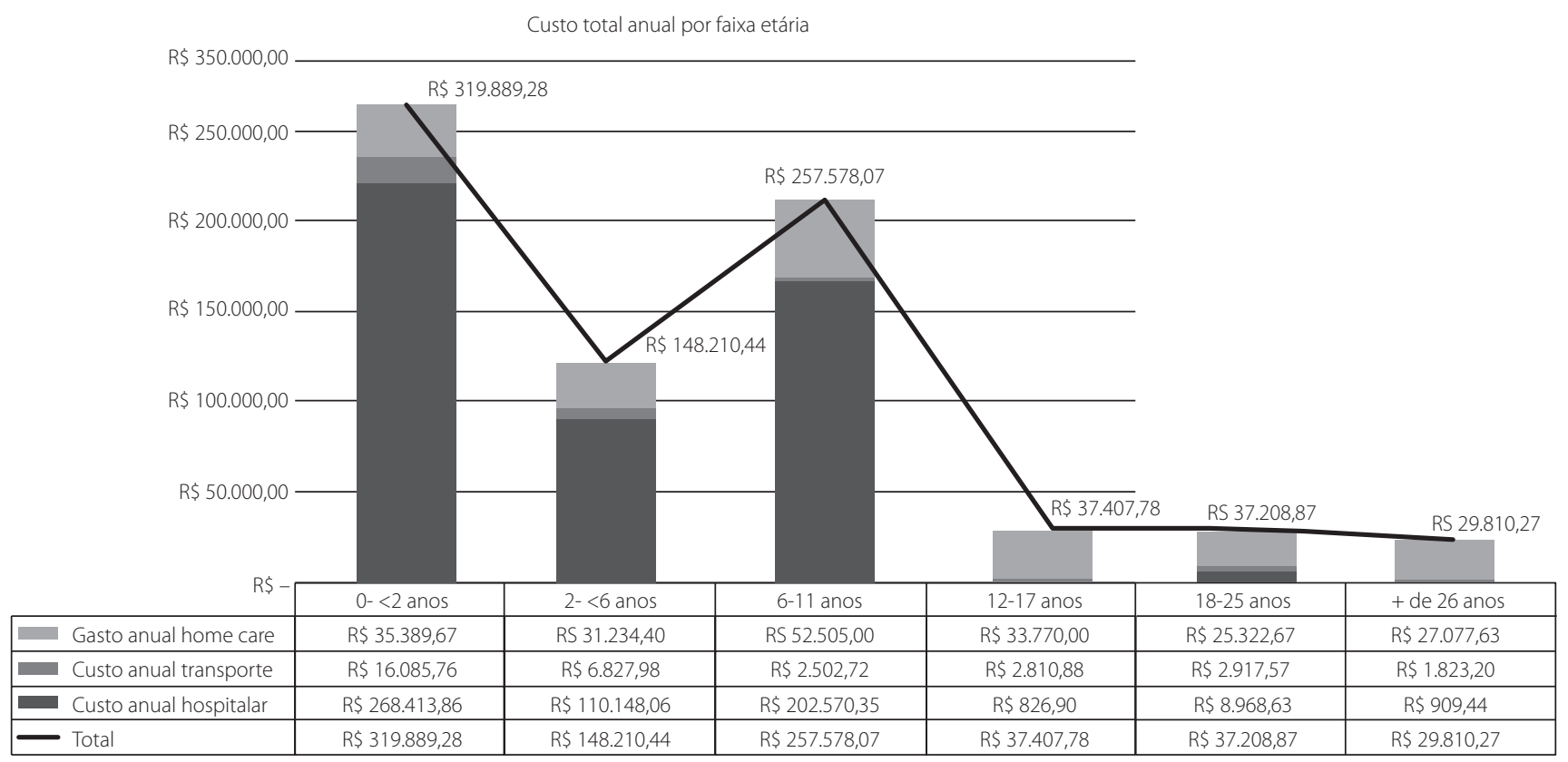

Figura 4. Custo total por faixa de idade.

\section{Discussão}

Estudos de microcusteio que avaliaram o custo do tratamento de pacientes com AME- $5 q$ são desconhecidos, de modo que a análise apresentada possui uma contribuição científica que pode subsidiar a tomada de decisão e planejamento da gestão de recursos de saúde, especialmente para o Brasil. Foi possível retratar um maior custo com pacientes nas faixas etárias de 0-2 anos e com AME-5q tipo 1 ou 2 e, também, um gasto importante com cuidados domiciliares, principalmente relacionados com a perda de produtividade de algum membro da família.
O custo hospitalar elevado com pacientes mais jovens portadores de AME-5q tipo 1 também foi reportado por um estudo que estimou o gasto anual com o tratamento da AME-5q por paciente a partir de relatórios de operadoras de saúde dos Estados Unidos. Internações hospitalares foram a maior parcela de custos na coorte de bebês (0-2 anos), enquanto custos com medicamentos contribuíram mais para custos totais na coorte de início na infância (Tan et al., 2019). Estudo também realizado nos Estados Unidos e que considerou a base de dados de pagamentos do sistema de saúde das forças militares norte-americanas identificou que a maior 
parcela das despesas em uma coorte de 239 pacientes diagnosticados com AME-5q era justificada por cuidados ambulatoriais (Armstrong et al., 2016). O estudo também avaliou que a mediana das despesas anuais com os cuidados da doença dos pacientes diagnosticados com menos de 1 ano $(n=45)$ é duas vezes superior à mediana da amostra estudada, que contempla pacientes com diagnóstico após 1 ano de idade $(n=194)$ (Armstrong et al., 2016).

Os resultados de custos hospitalares maiores do que aqueles incorridos pelas famílias em nosso estudo divergem do que foi apresentado em um estudo espanhol que também estimou o custo anual com o tratamento da doença, mas utilizou somente entrevistas com os familiares para a avaliação de custos associados ao tratamento da AME-5q. Na amostra espanhola, os entrevistados reportaram que $32,2 \%$ dos custos são atribuídos a custos hospitalares, e os custos de cuidados familiares representaram $62,7 \%$ do custo total de doença (López-Bastida et al., 2017). As diferenças metodológicas entre os estudos (o estudo espanhol utilizou apenas entrevistas com as famílias) e as diferenças entre a renda média familiar entre os países limitam a comparação entre os estudos.

As despesas anuais por paciente reportadas na literatura, coletadas com sistemas de remuneração e ou contas hospitalares, variam de € 6.274, na Espanha (Darbà \& Marsà, 2019), US\$29.988 e US\$306.204 (Tan et al., 2019), nos Estados Unidos, e $€ 70.4600$, na Alemanha (Klug et al., 2016). No presente estudo, a média global de custos medidos, independentemente da terapia utilizada ou da idade, foi de R\$ 101.516 ( $€ 23.071$ ou US\$ 25.765)'; desses, 32\% representam gastos domiciliares. Entretanto, é importante destacar que a comparação dos resultados entre o estudo apresentado nesse artigo, o estudo espanhol e o estudo norte-americano não pode ser feita de forma direta, na medida em que a metodologia de custeio foi heterogênea entre os estudos.

Apesar de os estudos usarem diferentes métodos para avaliar a carga econômica da doença, é ponto comum a elevada variabilidade de custos ou despesas entre os pacientes analisados. O presente estudo apresentou variabilidade importante mesmo dentro dos grupos e entre os fenótipos da doença e faixas de idade. A possível justificativa para essa variabilidade pode estar relacionada à gravidade da doença e às diferentes estratégias terapêuticas utilizadas no tratamento dos pacientes (Darbà \& Marsà, 2019). No presente estudo, para o grupo de pacientes em uso de nusinersena, o que contribuiu para a variabilidade foi o número de doses que cada paciente recebeu em função das diferentes datas de início do uso do medicamento.

1 Cotação média anual entre as moedas real (R\$) e dólar americano (US\$) em 2019 (3,94) e entre real (R\$) e euro (€) em $2019(4,40)$, de acordo com os dados do Instituto de Pesquisa Econômica Aplicada (Ipea).
Este estudo apresenta algumas limitações que devem ser consideradas para a interpretação dos resultados. Trata-se de análise de uma coorte de pacientes com AME-5q acompanhados em um único hospital-escola público brasileiro, por isso a generalização dos resultados é limitada. A coleta dos dados de consumo e recursos hospitalares deste estudo foi realizada de forma retrospectiva, a partir da revisão de prontuários eletrônicos, de forma que a qualidade dos dados é dependente do registro dos profissionais sobre os recursos utilizados. Para a estimativa de custos das famílias com os cuidados da doença, assume-se que pode haver viés de memória do entrevistado sobre o gasto decorrente da doença, e não foram considerados auxílios que o governo brasileiro concede para algumas famílias como suporte aos cuidados de doenças raras. Por fim, no que diz respeito ao gasto com transporte, foi estimado o mínimo que se entende ser gasto, baseado em um custo por quilômetro rodado e passagens urbanas municipais. Sabe-se que, na realidade do sistema de saúde brasileiro, esse gasto pode ser mais expressivo quando, por exemplo, municípios trazem os pacientes com motorista ao centro de referência.

\section{Conclusões}

O custo do manejo de pacientes com AME-5q no Brasil demonstra valores expressivos tanto na perspectiva do sistema de saúde quanto na das famílias dos pacientes. O estudo demonstrou que essas estimativas são diretamente relacionadas ao tipo da doença e ao uso de terapia específica. Cabe ressaltar que o estudo não fez análises relacionadas à eficácia ou ao benefício das terapias específicas e, dessa forma, não faz nenhuma menção ao custo-efetividade de tais intervenções.

$\mathrm{Na}$ abordagem única de microcusteio utilizada, foram identificadas algumas oportunidades para buscar reduzir o impacto dessa doença, bem como para aumentar a economicidade da terapia. Os custos com deslocamentos para as terapias e acompanhamento não são desprezíveis, tendo sido provavelmente subestimados na perspectiva da família e sociedade como um todo. A busca por estratégias que proporcionem levar o cuidado multidisciplinar ao paciente, de forma presencial ou remota, poderia ser perseguida.

Além disso, o impacto econômico da improdutividade, podendo também ser acrescida a essa análise a descontinuidade do trabalho dos familiares ao longo da vida, é bastante substancial. Quaisquer estimativas de custo-efetividade de terapias que melhorem a funcionalidade desses pacientes e reduzam o seu grau de dependência devem considerar esse custo indireto.

Por fim, a ampliação da amostra estudada, principalmente de pacientes que fizeram uso do nusinersena e que iniciarão o uso de novas terapias específicas recentemente aprovadas para tratamento da AME-5q, permitirá o alcance 
de estimativas mais robustas de custos com o cuidado da doença no Brasil e seu impacto na vida das famílias. Esses resultados, se somados aos desfechos clínicos esperados para as terapias, publicados na literatura, podem ser utilizados para o desenvolvimento de estudos de custo-efetividade que retratem a realidade econômica brasileira para subsidiar a tomada de decisão sobre a incorporação das tecnologias em saúde.

\section{Agradecimentos}

Agradecemos a pesquisadora Renata Ruschel, pelo trabalho de apoio à coleta de dados e, a pesquisadora Fabiana Casarin, pela colaboração com a revisão do conteúdo gerado.

\section{Referências bibliográficas}

Araújo AP, Ramos VG, Cabello PH. Dificuldades diagnósticas na atrofia muscular espinhal. Arq Neuro-Psiquiatr. 2005;63(1):145-9.

Armstrong EP, Malone DC, Yeh WS, Dahl GJ, Lee RL, Sicignano N. The economic burden of spinal muscular atrophy. J Med Econ. 2016;19(8):822-6.

Arnold WD, Kassar D, Kissel JT. Spinal muscular atrophy: Diagnosis and management in a new therapeutic era: Spinal Muscular Atrophy. Muscle Nerve. 2015;51(2):157-67.

Brasil. Ministério da Saúde. Protocolo Clínico e Diretrizes Terapêuticas da Atrofia Muscular Espinhal 5q tipo I. Brasília, DF: Ministério da Saúde; 2019.

Darbà J, Marsà A. Patient characteristics and hospitalisation costs of spinal muscular atrophy in Spain: a retrospective multicentre database analysis. BMJ Open. 2019;9(11):e031271.

Darras BT. Spinal Muscular Atrophies. Pediatr Clin North Am. 2015;62(3):743-66.

Etges APBS, Cruz LN, Notti RK, Neyeloff JL, Schlatter RP, Astigarraga CC, et al. An 8-step framework for implementing time-driven activity-based costing in healthcare studies. Eur J Health Econ. 2019b;20(8):1133-45.

Etges APBS, Ruschel KB, Polanczyk CA, Urman RD. Advances in ValueBased Healthcare by the Application of Time-Driven Activity-Based Costing for Inpatient Management: A Systematic Review. Value Health. 2020;23(6):812-23.
Etges APBS, Schlatter R, Neyeloff J, Araújo DV, Bahia L, Vruz L, et al. Estudos de Microcusteio aplicados a avaliações econômicas em saúde: uma proposta metodológica para o Brasil. J Bras Econom Saúde. 2019a;11(1).

Farrar MA, Carey KA, Paguinto SG, Chambers G, Kasparian NA. Financial, opportunity and psychosocial costs of spinal muscular atrophy: an exploratory qualitative analysis of Australian carer perspectives. BMJ Open. 2018;8(5):e020907.

Jeppesen J, Madsen A, Marquardt J, Rahbek J. Living and ageing with spinal muscular atrophy type 2: Observations among an unexplored patient population. Dev Neurorehabil. 2010;13(1):10-8.

Keel G, Savage C, Rafiq M, Mazzocato P. Time-driven activity-based costing in health care: A systematic review of the literature. Health Policy. 2017;121(7):755-63.

Klug C, Schreiber-Katz O, Thiele S, Schorling E, Zowe J, Reilich P, et al. Disease burden of spinal muscular atrophy in Germany. Orphanet J Rare Dis. 2016;11(1):58.

Liewluck T, Saperstein DS. Progressive muscular atrophy. Neurol Clin. 2015;33(4):761-73.

López-Bastida J, Peña-Longobardo LM, Aranda-Reneo I, Tizzano E, Sefton M, Oliva-Moreno J. Social/economic costs and health-related quality of life in patients with spinal muscular atrophy (SMA) in Spain. Orphanet J Rare Dis. 2017;12(1):141.

Lunn MR, Wang CH. Spinal muscular atrophy. Lancet. 2008;371(9630):2120-33.

Mercuri E, Finkel RS, Muntoni F, Wirth B, Montes J, Main M, et al. Diagnosis and management of spinal muscular atrophy: Part 1: Recommendations for diagnosis, rehabilitation, orthopedic and nutritional care. Neuromuscul Disord. 2018;28(2):103-15.

Tan H, Gu T, Chen E, Punekar R, Shieh PB. Healthcare Utilization, Costs of Care, and Mortality Among Psatients With Spinal Muscular Atrophy. J Health Econ Outcomes Res. 2019;6(3):185-95.

Tan SS, Rutten FFH, van Ineveld BM, Redekop WK, Hakkaart-van Roijen L. Comparing methodologies for the cost estimation of hospital services. Eur J Health Econ. 2009;10(1):39-45.

Verhaart IEC, Robertson A, Wilson IJ, Aartsma-Rus A, Cameron S, Jones CC, et al. Prevalence, incidence and carrier frequency of $5 q$-linked spinal muscular atrophy - a literature review. Orphanet J Rare Dis. 2017;12(1):124. 


\section{Anexos}

Anexo I. Entrevistas com familiares para estimativa do custo domiciliar

\section{Questionário para estimar o gasto mensal familiar com a doença:}

Este questionário pode ser respondido pelo portador da doença ou responsável pelo paciente portador da doença.

As respostas objetivam estimar o gasto mensal relacionado à doença da família do paciente.

Sob hipótese alguma as respostas serão compartilhadas com qualquer identificação.

1 - Em razão da $\mathrm{AME}$, qual o seu gasto mensal com medicamentos?

2 - O paciente com AME possui rotina de consultas com profissionais da saúde? Se sim, qual a frequência e o gasto mensal em consultas em razão da AME? (Listar todos os profissionais. Abaixo alguns exemplos.)

\section{Fisioterapia}

Número de consultas mensais:

Gasto mensal (R\$):

Nutricionista

Número de consultas mensais

Gasto mensal (R\$):

Psicólogo

Número de consultas mensais

Gasto mensal (R\$):

3 - Algum familiar deixou de trabalhar para dedicar cuidados ao filho com AME? Se sim, qual era a renda mensal aproximada desse familiar?

4 - A família arca com algum tipo de cuidador para auxiliar nos cuidados com o paciente com AME? Se sim, qual valor mensal (em reais)?

5 - A família precisou fazer algum investimento na infraestrutura da moradia para atender às necessidades do paciente com AME? Se sim, qual? Quanto (em reais)?

(Por exemplo, cama especial, cadeira de rodas, equipamento para auxiliar na alimentação etc.)

Anexo II. Tempo de dedicação dos profissionais com o cuidado de pacientes com AME-5q

\begin{tabular}{|c|c|c|c|}
\hline Profissional & $\begin{array}{c}\text { Consulta em } \\
\text { ambulatório }(\mathrm{min})\end{array}$ & Cuidados na rotina de internação (min) & $\begin{array}{l}\text { Acompanhamento de residente da } \\
\text { especialidade }\end{array}$ \\
\hline Médico pediatra & $60 \mathrm{~min}$ & $\begin{array}{l}30 \text { min por dia (se paciente internado na UI) } \\
60 \text { min por dia (se paciente internado na UTI) }\end{array}$ & Em todos os atendimentos \\
\hline Médico geneticista & $60 \mathrm{~min}$ & $\begin{array}{c}1 \mathrm{a}, 2^{\mathrm{a}} \text { consultorias } 60 \mathrm{~min} \\
\text { Demais consultorias } 15 \mathrm{~min}\end{array}$ & Em todos os atendimentos \\
\hline Fisioterapeuta & Na fisiatria: $120 \mathrm{~min}$ & 45 min por atendimento & Na primeira semana de internação \\
\hline Terapeuta ocupacional & Na fisiatria: $120 \mathrm{~min}$ & - & - \\
\hline Médico fisiatra & $60 \mathrm{~min}$ & - & Em todos os atendimentos \\
\hline Enfermagem & 30 min & 120 min por dia sem intercorrência & Independente para atendimento \\
\hline Técnico de enfermagem & - & 5 horas por dia & - \\
\hline Médico pneumologista & $60 \mathrm{~min}$ & $\begin{array}{l}1^{\text {a }} \text { consultoria } 120 \mathrm{~min} \\
\text { Demais consultorias } 20 \mathrm{~min}\end{array}$ & Em todos os atendimentos \\
\hline Fonoaudiólogo & - & $\begin{array}{l}1^{\text {a }} \text { consultoria } 60 \mathrm{~min} \\
\text { Demais consultorias } 40 \text { min por } \\
\text { atendimento }\end{array}$ & Em todos os atendimentos \\
\hline Nutricionista & - & $\begin{array}{l}\text { 1a consultoria } 90 \mathrm{~min} \\
\text { Demais consultorias } 40 \text { min por } \\
\text { atendimento } \\
\text { Orientação alta } 60 \mathrm{~min}\end{array}$ & Em todos os atendimentos \\
\hline Psicólogo & - & 30 min discussão de caso com residente & $\begin{array}{c}50 \text { a } 60 \text { min por atendimento } 30 \text { min } \\
\text { discussão de caso uma vez por semana } \\
\text { com preceptor } \\
\text { Orientação alta } 20 \text { min }\end{array}$ \\
\hline Médico intensivista & - & 60 min por dia & Em todos os atendimentos \\
\hline Demais médicos & - & 30 min por dia & Em todos os atendimentos \\
\hline
\end{tabular}

UI: unidade de internação; UTI: unidade de tratamento intensivo. 
Anexo III. Detalhamento do custo com transporte, estratificado por tipo de AME e faixa etária
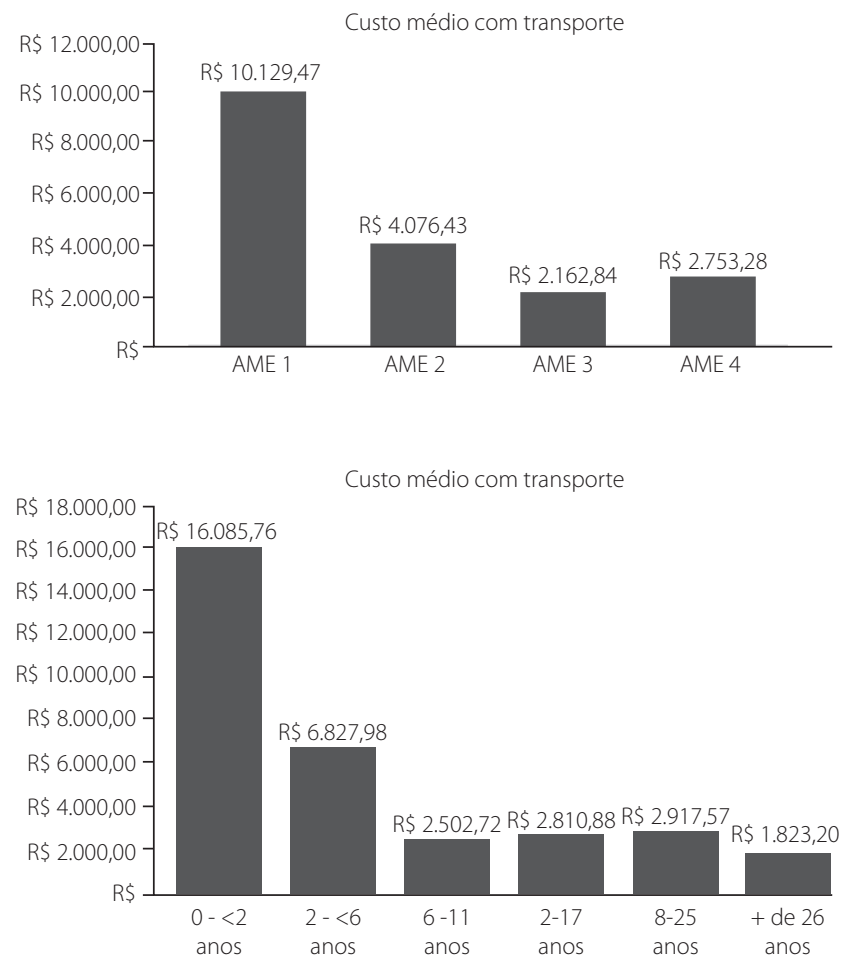

Anexo IV. Custo total por paciente por tipo de fenótipo da doença e faixa etária

\begin{tabular}{|c|c|c|c|}
\hline Tipo ou faixa etária & Custo anual hospitalar & Custo anual com transporte & $\begin{array}{c}\text { Gasto anual com cuidados } \\
\text { domiciliares }\end{array}$ \\
\hline AME 1 & $\begin{array}{c}\text { Média } R \$ 147.754 \\
(0-802.566) \\
\text { Med R\$1.350 }\end{array}$ & $\begin{array}{c}\text { Média } R \$ 10.129 \\
\text { (54-73.382) } \\
\text { Med } R \$ 2.828\end{array}$ & $\begin{array}{c}\text { Média } R \$ 34.298 \\
\quad(0-163.480) \\
\text { Med } R \$ 18.000\end{array}$ \\
\hline AME 2 & $\begin{array}{c}\text { Média R\$101.899 } \\
\text { (190-845.629) } \\
\text { Med R\$ 1.152 }\end{array}$ & $\begin{array}{c}\text { Média R\$ } 4.076 \\
(36-23.846) \\
\text { Med } R \$ 2.476\end{array}$ & $\begin{array}{c}\text { Média } R \$ 42.261 \\
(0-193.176) \\
\text { Med } R \$ 8.004\end{array}$ \\
\hline AME 3 & $\begin{array}{c}\text { Média } R \$ 1.478 \\
\text { (304-10.485) } \\
\text { Med } R \$ 914\end{array}$ & $\begin{array}{c}\text { Média R\$2.162 } \\
(18-9.331) \\
\text { Med R\$ } 1.497\end{array}$ & $\begin{array}{c}\text { Média } R \$ 16.098 \\
\quad(0-69.696) \\
\text { Med } R \$ 32.364\end{array}$ \\
\hline AME 4 & $\begin{array}{c}\text { Média } R \$ 1.221 \\
(1.161-1.280) \\
\text { Med } R \$ 1.221\end{array}$ & $\begin{array}{c}\text { Média } R \$ 2.753 \\
(18-9.331) \\
\text { Med } R \$ 2.753\end{array}$ & $\begin{array}{c}\text { Média } R \$ 32.364 \\
(32.364-32.364) \\
\text { Med } R \$ 32.364\end{array}$ \\
\hline $0-<2$ anos & $\begin{array}{c}\text { Média } R \$ 268.413 \\
(0-802.566) \\
\text { Med } R \$ 190.757\end{array}$ & $\begin{array}{c}\text { Média } R \$ 16.085 \\
\text { (293-73.846) } \\
\text { Med R\$ } 4.392\end{array}$ & $\begin{array}{c}\text { Média } R \$ 35.389 \\
(0-109.084) \\
\text { Med } R \$ 14.901\end{array}$ \\
\hline $2-<6$ anos & $\begin{array}{c}\text { Média R\$ } 110.148 \\
\text { (437-649.194) } \\
\text { Med R\$ } 2.158\end{array}$ & $\begin{array}{c}\text { Média } R \$ 6.827 \\
\quad(54-23.846) \\
\text { Med } R \$ 3.090 \\
\end{array}$ & $\begin{array}{c}\text { Média } R \$ 31.234 \\
(11.976-86.436) \\
\text { Med } R \$ 16.800\end{array}$ \\
\hline 6-11 anos & $\begin{array}{c}\text { Média } R \$ 202.570 \\
\text { (945-806.069) } \\
\text { Med } R \$ 1.633 \\
\end{array}$ & $\begin{array}{c}\text { Média R\$2.502 } \\
(622-4.651) \\
\text { Med } R \$ 2.413 \\
\end{array}$ & $\begin{array}{c}\text { Média } R \$ 52.505 \\
(15.600-124.100) \\
\text { Med } R \$ 35.160\end{array}$ \\
\hline 12-17 anos & $\begin{array}{c}\text { Média R\$ } 826 \\
(190-1.751) \\
\text { Med } R \$ 682\end{array}$ & $\begin{array}{c}\text { Média R\$2.810 } \\
(380-5.656) \\
\text { Med } R \$ 2.603\end{array}$ & $\begin{array}{c}\text { Média } R \$ 33.770 \\
(33.700-124.100) \\
\text { Med } R \$ 33.770\end{array}$ \\
\hline $18-25$ anos & $\begin{array}{c}\text { Média R\$ } 8.968 \\
(304-64.854) \\
\text { Med R\$ } 1.146 \\
\end{array}$ & $\begin{array}{c}\text { Média R\$ } 2.917 \\
(36-10.857) \\
\text { Med } R \$ 1.641\end{array}$ & $\begin{array}{c}\text { Média } R \$ 25.322 \\
(0-137.952) \\
\text { Med } R \$ 440\end{array}$ \\
\hline+ de 26 anos & $\begin{array}{c}\text { Média R\$ } 909 \\
(304-2.296) \\
\text { Med } R \$ 794\end{array}$ & $\begin{array}{c}\text { Média } R \$ 1.823 \\
(18-9.331) \\
\text { Med } R \$ 593\end{array}$ & $\begin{array}{c}\text { Média } R \$ 27.077 \\
(2.000-110.136) \\
\text { Med } R \$ 9.810\end{array}$ \\
\hline
\end{tabular}

Dados apresentados como média, valores mínimos e máximos e mediana. 
Anexo V. Custo anual hospitalar por fenótipo AME-5q para pacientes qua não fizeram uso do medicamento

\begin{tabular}{|c|c|c|c|c|c|c|}
\hline Tipo & $\mathrm{N}$ & Custo ambulatorial & Custo internação & Custos exames & $\begin{array}{l}\text { Custo materiais e } \\
\text { medicamentos }\end{array}$ & Custo anual \\
\hline AME 1 & 8 & $\begin{array}{c}\text { Média R\$ } 1.719 \\
(437-6.323) \\
\text { Med R\$ } 1.181\end{array}$ & $\begin{array}{c}\text { Média R\$ } 8.453 \\
(0-57.689) \\
\text { Med R\$ } 123\end{array}$ & $\begin{array}{c}\text { Média R\$ } 41 \\
(0-330) \\
\text { Med R\$ } 66\end{array}$ & $\begin{array}{c}\text { Média R\$ } 357 \\
(0-2.794) \\
\text { Med R\$ - }\end{array}$ & $\begin{array}{c}\text { Média } R \$ 10.571 \\
(437-67.138) \\
\text { Med R\$1.304 }\end{array}$ \\
\hline AME 2 & 13 & $\begin{array}{c}\text { Média R\$ } 1.297 \\
(190-5.842) \\
\text { Med R\$ } 945\end{array}$ & $\begin{array}{c}\text { Média R\$3.343 } \\
\text { (0-40.119) } \\
\text { Med R\$ - }\end{array}$ & $\begin{array}{c}\text { Média R\$ } 66 \\
\text { (0-866) } \\
\text { Med R\$ - }\end{array}$ & $\begin{array}{c}\text { Média R\$ } 1.386 \\
\text { (0-18.026) } \\
\text { Med R\$ - }\end{array}$ & $\begin{array}{c}\text { Média } R \$ 6.093 \\
(190-64.854) \\
\text { Med R\$ } 945\end{array}$ \\
\hline AME 3 & 15 & $\begin{array}{c}\text { Média R\$932 } \\
(304-2.296) \\
\text { Med R\$ } 914\end{array}$ & $\begin{array}{c}\text { Média } R \$ 550 \\
(0-7.703) \\
\text { Med } R \$ 0\end{array}$ & $\begin{array}{c}\text { Média R\$ } 4 \\
(0-69) \\
\text { Med R\$ } 0\end{array}$ & $\begin{array}{c}\text { Média R\$ } 0 \\
(0-416) \\
\text { Med R\$̦ } 0\end{array}$ & $\begin{array}{c}\text { Média R\$1.515 } \\
\text { (304-10.485) } \\
\text { Med R\$914 }\end{array}$ \\
\hline AME 4 & 2 & $\begin{array}{c}\text { Média R\$1.221 } \\
(1.161-1.280) \\
\text { Med R\$ } 1.221\end{array}$ & $\mathrm{R} \$-$ & $\mathrm{R} \$-$ & $\begin{array}{l}\mathrm{R} \$- \\
\mathrm{R} \$-\end{array}$ & $\begin{array}{c}\text { Média } R \$ 1.221 \\
(1.161-1280) \\
\text { Med } R \$ 1.221\end{array}$ \\
\hline
\end{tabular}

A tabela apresenta os valores médios (intervalo mínino e máximo) e medianas por item de custo e tipo de AME-5q. Med: mediana. 